
\title{
3 Research Square \\ The Effect of Glutamine Supplementation on Serum Levels of Some Inflammatory Factors, Oxidative Stress, and Appetite in COVID-19 Patients: A Case- Control Study
}

mahsa mohajeri ( $\nabla$ mahsa.mohajeri.93@gmail.com )

Ardabil University of Medical Sciences: Ardebil University of Medical Sciences https://orcid.org/00000001-5156-7398

ehsan horriatkhah

Baku State University

\section{Research Article}

Keywords: glutamine, COVID-19, appetite, oxidative stress

Posted Date: July 30th, 2021

DOl: https://doi.org/10.21203/rs.3.rs-748690/v1

License: (c) (1) This work is licensed under a Creative Commons Attribution 4.0 International License.

Read Full License

Version of Record: A version of this preprint was published at Inflammopharmacology on October 28th, 2021. See the published version at https://doi.org/10.1007/s10787-021-00881-0. 


\section{Abstract}

Background: Malnutrition is seen in COVID-19 patients, and reducing malnutrition with appropriate therapies may improve these patients' health. this case-control study aimed to assess and compare serum levels of some inflammatory factors and oxidative stress and appetite in COVID-19 patients with respiratory infections that receive glutamine treatment with a control group.

Method: In this study, patients who consented to use glutamine were considered as the case group and other patients who did not use glutamine were considered as a control group. Twenty-seven Covid-19 patients (10 female, $48.5 \pm 9.1$ ) using L-Glutamine and 27 Covid-19 patients (12 female, $48.8 \pm 4.6$ ) with similar age, gender, and clinical status were included in the study. For five days, the case group was given $10 \mathrm{~g} /$ day of glutamine supplement. At the end of the fifth day, blood samples were taken again to test for serum levels of IL $1 \beta$, tumor necrosis factor-alpha, malondialdehyde, and total antioxidant capacity, then all data were analyzed.

Results: Serum levels of beta-1 interleukin, tumor necrosis factor-alpha, and hs-CRP were significantly reduced with five days of glutamine supplementation $(p<0.05)$, and patients' appetite during five days of glutamine supplementation compared with the control group had a significant increase $(p<0.05$.

Conclusion: Glutamine supplementation in COVID-19 patients with respiratory infection significantly reduces serum levels of interleukin- 1 beta, hs-CRP, and tumor necrosis factor-alpha and significantly increases appetite, so glutamine supplementation may be useful for COVID-19 patients in the hospital.

\section{Introduction}

On March 11, 2020, the World Health Organization declared the Covid-19 as a pandemic (Daniel, 2020). The main approach, especially in those with lower respiratory tract involvement, is focused on optimizing respiratory functions. In the elderly patients with low immune function, in individuals with nutritional deficiencies, and people with chronic diseases the morbidity and mortality of the COVID-19 is higher (Liu et al., 2020; Paladugu \& Donato, 2020). In COVID-19, the most common complication and the problem is related to respiratory disease. Since the catabolic process continues in this disease normal nutrition intake is not sufficient to support recovery. Decreasing the acute stress levels of specific amino acids, such as and glutamine enhances immunity in these patients (Naja \& Hamadeh, 2020). Various treatments are offered to improve respiratory infections, along with treatment, several supplements are used. One of the supplements used is the amino acid glutamine. Glutamine is the most abundant amino acid in plasma and skeletal muscle and contains more than $60 \%$ of all intramuscular free amino acids (Cruzat, Macedo Rogero, Noel Keane, Curi, \& Newsholme, 2018). Glutamine is a precursor to the synthesis of amino acids, proteins, nucleotides, and many other biological molecules (6). Glutamine is largely synthesized in muscle and is used for gluconeogenesis in the liver (Cruzat et al., 2018; Shah, Wang, \& Ma, 2020). It is an important fuel for the cells of the immune system and may have stimulating effects on the immune system (Andersen et al., 2019). Glutamine also has antioxidant properties and increasing the 
body's glutathione reserves can increase the body's antioxidant capacity (Moura, Lollo, Morato, Risso, \& Amaya-Farfan, 2017) and reduce oxidative stress and inflammatory factors. It has been shown that a daily dose of $0.5 \mathrm{~g} / \mathrm{kg}$ of glutamine supplementation reduces nosocomial infections in hospitalized patients (Stehle et al., 2017). Some studies have shown that glutamine regulates the immune system in animals and humans (Cruzat et al., 2018). Another study showed that intravenous glutamine supplementation for 5 days can reduce the frequency of pneumonia, infection, and bacteremia in patients with multiple trauma (Stehle et al., 2017). Malnutrition is seen in COVID-19 patients, and reducing malnutrition with appropriate therapies may improve these patient's health (Anker et al., 2020). Malnutrition also exacerbates infection and increases mortality in COVID-19 by weakening the immune system (16). In some animal studies, it has been shown that infection reduces appetite, and eventually, the animal loses weight (T. Li et al., 2020). Possible mechanisms of malnutrition in infectious patients include increased inflammatory factors such as elevated serum CRP levels (Derouiche, 2020) and inflammatory interleukins such as IL1, IL6, and a-TNF. Numerous studies show that serum inflammatory factors (interleukins such as IL6, IL1 $\beta$, and TNF- $\alpha$ ) are elevated in these patients (Robinson et al., 2020). IL1 $1 \beta$ strongly promotes anorexia, increased energy expenditure, muscle protein loss, and leptin release (Rao et al., 2017). Oxidative stress also exacerbates malnutrition in these patients through changes in metabolism and energy (Hsieh, Yang, \& Tsai, 2016). It has been reported that nutritional support in terms of energy and nutrient supply can improve nutritional status and anthropometric factors in patients with respiratory infections (Baumgartner et al., 2021). On the other hand, it has been shown that glutamine can regulate appetite by affecting the secretion of Glucagon-like peptide 1 from the gastrointestinal tract (Andersson et al., 2018). Glucagon-like peptide 1 is a physiological regulator of energy intake and appetite (Adams et al., 2018). Glutamine is also involved in the formation of glutamate and GABA (Qureshi et al., 2020)and glutamate and GABA have been shown to stimulate appetite (Varela et al., 2021). Glutamine has the effect of reducing pro-inflammatory cytokines and may play a role in reducing mortality by controlling the infection (de Urbina et al., 2017). Only one study (Cengiz et al., 2020) investigated the glutamine supplementation effect on COVID-19 treatment and its result indicated that adding enteral L-glutamine to the normal nutrition in the early period of COVID-19 infection may lead to a shortened hospital stay and lead to less need for ICU, and until now there is no study about the glutamine supplementation effect on COVID-19 patients' serum levels of some inflammatory factors, oxidative stress, and appetite. Based on the COVID-19 treatment importance and reduction in mortality and morbidity, this case-control study aimed to assess and compare serum levels of some inflammatory factors and oxidative stress and appetite in COVID-19 patients with respiratory infections that receive glutamine treatment with a control group.

\section{Methods}

\section{Study population}

A total of 284 Covid- 19 patients were screened and 54 patients who met the inclusion criteria were included in the study. Patients who applied to the Covid-19 outpatient clinics of Baku University Hospital 
between Jan 28 and Mar 10, those who had lower respiratory tract involvement in computed thorax tomography (thorax CT) and positive real-time reverse-transcriptase-polymerase chain reaction (RT-PCR) test in oro-nasopharyngeal swab were included in the study. Glutamine supplementation is routinely ordered by a physician in the intensive care unit with the consent of the patient or his family and is purchased and used by the patient's family. In this study, patients who consented to use glutamine were considered as the case group and other patients who did not use glutamine were considered as a control group. Twenty-seven Covid-19 patients (10 female, $48.5 \pm 9.1$ ) using L-Glutamine and 27 Covid-19 patients (12 female, $48.8 \pm 4.6$ ) with similar age, gender, and clinical status were included in the study.

Patients with kidney and liver dysfunction, alcoholism, malignancy, connective tissue diseases, cardiovascular diseases, diabetes mellitus, neurological and psychiatric problems (Parkinson's disease, cerebrovascular disease, delirium, bipolar disorder, depression), or severe disease that cannot be measured their weight or height were excluded from the study. The Sequential Organ Failure Assessment (SOFA) scoring was performed on all patients for evaluating the severity of the disease before the beginning of the study. The patients who had a point of quick SOFA (qSOFA) _2 were also not included in the study. When a patient was classified with or at risk of malnutrition at the beginning or in the following period of the hospital stay, we planned a nutritional care plan and excluded them from the study. The patients whose clinical courses and laboratory parameters worsened through all given treatments were evaluated as severe sepsis according to the criteria of SOFA. Firstly qSOFA was calculated with the blood pressure, respiratory rate, and mental status, if the qSOFA is 2 and/or bigger than 2, the patient was taken to the ICU. SOFA measures individual or aggregate organ dysfunction in six organ systems (respiratory, coagulatory, liver, cardiovascular, renal, and neurologic) in the ICU and mostly predict hospital mortality.

Diagnosis of Covid-19

Thorax CT screenings of all patients were taken at the time of hospital admission. As stated in the guidelines of the Azerbaijan Ministry of Health, an oropharyngeal sample was first taken with a swab, then a nasal sample was taken using the same swab, and placed in the same transport medium for diagnosis. Samples were tested by RT-PCR assay developed from the virus sequence.

\section{Laboratory analysis}

Before giving the supplement, $2 \mathrm{cc}$ blood samples were taken from both groups. Then for five days, the case group was given a glutamine supplement at the rate of $0.5 \mathrm{~g} / \mathrm{kg}$ with a tablespoon of sugar, and the control group was given the same amount of sugar. At the end of the fifth day, blood samples were taken again to test for serum levels of IL1 $\beta$, tumor necrosis factor-alpha, malondialdehyde, and total antioxidant and malondialdehyde capacity, then all data were analyzed. COVID-19 patients with certain diseases, such as heart and liver disease, and those who were unwilling to cooperate were excluded from the study.

To measure this cytokine, the sandwich ELISA method was used using the human ELISA kit manufactured by Diaclone Company with serial number 950.090.096. 
To measure the total antioxidant capacity, RANDOX's RANSOM kit was used according to the recommended protocol by spectrophotometry using an Abbot autoanalyzer at $600 \mathrm{~nm}$. The basis of this measurement is based on the incubation of a special substrate with peroxidase and hydrogen peroxide and the production of a radical substrate cation that produces a stable green-blue color that can be measured at a wavelength of $600 \mathrm{~nm}$. The TAC value is in $\mathrm{mmol} / \mathrm{I}$.

To measure hs-CRP protein, Pars test diagnostic kit was used by immunoturbidimetric. According to the kit brochure, first $20 \mu \mathrm{l}$ of serum with $200 \mu \mathrm{l}$ of reagent 1 was placed at $37^{\circ} \mathrm{C}$ for 5 minutes, then $200 \mu \mathrm{l}$ of reagent 2 containing mouse monoclonal and goat polyclonal antibodies against human CRP antibody was added. Absorption was measured at 30 and 90 seconds with the Abbot autoanalyzer at $500 \mathrm{~nm}$ in $\mathrm{mg} / \mathrm{l}$.

1-beta interleukins were measured by ELISA using Bender Med Systems kits (Vienna, Austria).

After discharging the patients before 5 days before the hospitalization period, according to the present project, the necessary recommendations regarding the use of supplements and placebo were provided to the parents and the patients were followed up to control them in terms of taking supplements.

\section{Glutamine supplementation and nutritional status screening}

The patient's nutritional status was investigated with the Nutritional Risk Screening (NRS-2002) at the hospital admission time. NRS-2002 has two levels such as impaired nutritional status and severity of disease (such as low, moderate, or severe for both of them), with an adjustment for age _70 years. The nutrition status as considering the first level of this screening was evaluated by the variables; Body Mass Index (BMI), weight loss in the last 3 months, and decreased food intake in the last week. The severity degrees of disease as considering the second level of NRS-2002 was defined as absent, mild, moderate, or severe that was converted to a numeric score between 1 and 3 according to recommendations. A total score under 3 suggested no nutritional risk. To obtain all information a data collection sheet was used.

All given meals for this study patients were prepared based on the appropriate guidelines in COVID-19 and consisted of equal protein and calorie contents. To the case group, $10 \mathrm{~g} \mathrm{L-Glutamine} \mathrm{available} \mathrm{in}$ powder forms (Resource Glutamine, Nestle) were given 3 times a day with meals.

\section{Appetite}

In this study, a subjective index and an objective index for appetite measurement were considered. A visual analog scale (VAS) was used to quantify a subject's feelings on the desire to eat, hunger, prospective consumption, and fullness (for subjective parameters). To measure the food greasiness degree, food greasiness was added to the VAS. The scale 0-10 was adopted to evaluate the sensation before or after eating, and the higher the scale indicated the stronger the sensation. Based on their experience, the subjects were free to calibrate on each line that best matched how they were. 
This study is a case-control study for evaluating the glutamine effect on serum levels of some inflammatory factors and oxidative stress and appetite in COVID-19 patients with respiratory infections. A sample size of $n 1 / 429$ per group is required to provide $80 \%$ power to detect a difference in the mean levels with a significance of 0.05 (2-sided a).

\section{Statically analysis:}

The normal distribution of the data was tested using the one-sample Kolmogorov - Smirnov test. Continuous variables are presented as mean \pm standard deviation. Categorical variables are presented as counts. The statistical comparisons were performed using the two-sided Student's t-test. Categorical variables were compared using the Chi-square test or Fisher exact test for small samples. Values of $\mathrm{p}_{\text {_ }}$ 0.05 were considered to be statistically significant. The statistical analyses were performed using SPSS 20.0 software (SPSS, Chicago, IL, USA) for Windows.

\section{Results}

Demographic features of the study groups are given in Table 1. Age, gender distribution, and smoking were similar between the groups. Symptoms, medications for COVID-19, and physical examination findings of the study groups are given in Table 2. There was no significant difference in all of the symptoms, medications, and physical examination findings between the study groups.

Necessity of intensive care unit, duration of hospitalization, and the number of mortality of the groups of study are given in Table 3. Duration of hospitalization was found as $12.1 \pm 1.3$ days in the control group and $7.8 \pm 1.6$ days in the intervention group $(P=0.005)$. The number of the necessity of intensive care units was significantly higher in the control group $(p=0.018)$. There was a significant difference in mortality rates between the groups, 6 deaths were observed in the control group, which did not receive LGlutamine. Demographic characteristics, laboratory and physical examination findings of before and after the treatment of glutamine groups are given in Table 4 . In the case group, a significant decrease in total qSOFA score and respiratory rate was observed ( $p=0.015$ and $p=0.024$, respectively). In the case group after glutamine supplementation, all of the stress oxidative factors were significantly decreased and all of the serum antioxidant factors were significantly increased $(p<0.05)$. Table 5 indicated the appetite status of study participants. The results reported that in the case group the appetite was significantly improved $(p<0.05)$. According to Table 4 , the Chi-square test was used for appetite comparison before and after of study and the results showed that in the case group appetite increased significantly during the five days of glutamine supplementation compared to the control group $(p=0.02)$. After study in the case group the TNF-a ( $p=0.014)$, hs-CRP ( $P=0.011)$, MDA ( $p=0.015)$, IL-B1 ( $P=0.043)$ and TAC $(P=0.021)$ decreased significantly, these factors did not change significantly after the study.

\section{Discussion}


This study is the first study to evaluate the effect of glutamine on serum antioxidants, TNF-a, CRP, MDA, interleukin- $\beta_{1}$, and the level of appetite in COVID-19 patients admitted to the intensive care

unit. Decreased appetite has been reported in COVID-19 patients. This problem leads to a decrease in the level of immunity of the body and as a result, the negative effects of the virus on the person. Therefore, improving the appetite level in COVID-19 patients is a very important issue and it is recommended to use glutamine to improve the appetite of these patients. Decreased appetite leads to a low intake of nutrients effective in immunity such as vitamin C, zinc, selenium, and protein, and as a result, the complications of the disease in these people increase. The results of the present study showed that the amount of appetite during the five days of glutamine supplementation was significantly increased compared to the control group. Also in this present study results showed that the mean serum interleukin-1 beta and tumor necrosis factor-alpha were significantly reduced by taking a five-day glutamine supplement Increased levels of oxidative stress have been observed in patients with COVID-19 (Chernyak et al., 2020). Oxidative stress is responsible for the deterioration of these patients and cell damage (Laforge et al., 2020). Proper and healthy nutrition that is rich in antioxidants to increase serum antioxidant levels in these patients has a very important role in their recovery (Iddir et al., 2020). Glutamine is a supplement that is used as an antioxidant in some intensive care units of hospitals for critically ill patients, especially those with respiratory infections. (Cruzat et al., 2018). Skeletal muscle is the main reservoir of glutamine, and during infection the release of glutamine from the muscle doubles, so its serum level remains normal (Levitt \& Levitt, 2018). Cytokines and glucocorticoids increase the absorption of glutamine by the liver in infectious conditions (Cruzat et al., 2018). On the other hand, Cytokines regulate bowel movements and alter various brain signals involved in appetite (A. Patel, 2021)' IL1 $\beta$ strongly promotes anorexia, increased energy expenditure, muscle protein loss, and leptin release (Peixoto da Silva, Santos, Costa e Silva, Gil da Costa, \& Medeiros, 2020), TNF-a also causes anorexia, muscle protein breakdown, inhibition of lipoprotein lipase, and reduction of lipid production (H. J. Patel \& Patel, 2017) and is associated with pathogenesis, development, and progression of various infections, autoimmune diseases, malignant diseases, etc. . It has been shown that glutamine administration may play a role in improving the immune system (Cetinbas, Yelken, \& Gulbas, 2010), reducing pneumonia (Rogero \& dos Santos, 2017)and ultimately in reducing mortality in patients. Consistent with the results of a study by Fan et al (Fan et al., 2009) on animal models, showed that enteral and parenteral supplementation of glutamine significantly increased plasma and tissue glutamine concentrations and decreased TNF- $\alpha$ and IL $1 \beta$ plasma levels. Inconsistent with our result, the findings of the study by Doug et al. showed that glutamine supplementation for eight weeks did not affect inflammatory factors in the pulmonary sputum of cystic fibrosis patients that had a respiratory infection (Delgado, 2013). This different result may be related to the different types of studies sample.

The results of the present study showed that the serum level of hs-CRP was significantly reduced with five days of glutamine supplementation. Glutamine is a dietary supplement that has antioxidant properties (Yuan, Zhang, \& Li, 2017). In COVID-19 patients, the level of hs-CRP in the blood increases (Q. $\mathrm{Li}$ et al., 2020). Animal studies have shown that glutamine can improve the antioxidant capacity of the body by increasing glutathione stores (Yeh, Shih, \& Lin, 2020). Consistent with the findings of our study, 
Faqihzadeh et al.(F, P, \& A, 2014) in patients with non-alcoholic fatty liver disease, Tomé-Carneiro in patients with coronary artery disease(Tomé-Carneiro et al., 2013), and Bo et al. in smokers(Bo et al., 2013) showed that the use of some dietary antioxidants can reduce serum hs-CRP levels. The possible effect of glutamine in reducing serum hs-CRP may be due to its antioxidant properties, which can increase the activity of serum antioxidant enzymes (Tanha, Amanlo, \& Fathi, 2012) and ultimately control serum hs-CRP levels. In the present study, the serum level of TAC, in COVID-19 patients with glutamine treatment, was significantly increased by taking the glutamine supplement. Also, the patient's serum mean of MDA had significant changes after five glutamine supplementation. Increased oxidative stress is seen in patients with COVID-19, which may play a role in the pathogenesis of this disease. The end product of lipid peroxidation is active aldehydes such as malondialdehyde (MDA) (Tanha et al., 2012). Which can be used as a marker to measure the level of oxidative stress in the body. Oxidative stress occurs when free radical production is more than antioxidant capacity. Antioxidant enzymes and a variety of antioxidant compounds are produced in the body to counteract oxidative stress (Wang, Wang, Li, Lu, \& Liu, 2019) to protect cells and ultimately tissues from damage caused by free radicals and ROS; there is a significant change in the level of TAC and MDA after the use of glutamine in COVID- 19 with respiratory infections, reducing the amount TNF-a, hs-CRP and IL $B_{1}$ can be useful to improve the condition of these patients. The duration of the intervention, the small number of patients, the problems of preparing laboratory kits, and the low cooperation of the COVID-19 patient ${ }^{5 \prime}$ families were some of the limitations of this study. It is suggested that future studies be performed with more COVID-19 patients and with a longer intervention duration.

\section{Conclusion}

Glutamine supplementation at the rate of $10 \mathrm{~g} /$ day in COVID-19 patients with respiratory infections decreases the serum interleukin-beta level, the level of alpha necrosis tumor factor, the serum hs-CRP level and increases the appetite of COVID-19 patients, so using this supplement can prevent malnutrition of COVID-19 patients with respiratory infectious diseases.

\section{Declarations}

\section{Acknowledgment:}

The authors would like to thank Baku University, Ardabil University of Medical Sciences, and all the 19 patients who participated in this study.

\section{Conflict of interest:}

The authors of this article have no conflict of interest

\section{Informed consent}


This study was approved by the Ethics Committee of the Baku University Faculty of Medicine (ethical Number: 2020/4231). Before the written consent, all patients were given complete information about the study protocol.

\section{References}

1. Adams, J. M., Pei, H., Sandoval, D. A., Seeley, R. J., Chang, R. B., Liberles, S. D., \& Olson, D. P. (2018). Liraglutide Modulates Appetite and Body Weight Through Glucagon-Like Peptide 1 ReceptorExpressing Glutamatergic Neurons. Diabetes, 67(8), 1538-1548.

2. Andersen, A. D., Nguyen, D. N., Langhorn, L., Renes, I. B., van Elburg, R. M., Hartog, A., . . Thymann, T. (2019). Synbiotics combined with glutamine stimulate brain development and the immune system in preterm pigs. The Journal of nutrition, 149(1), 36-45.

3. Andersson, L. E., Shcherbina, L., Al-Majdoub, M., Vishnu, N., Arroyo, C. B., Carrara, J. A., . . Wierup, N. (2018). Glutamine-elicited secretion of glucagon-like peptide 1 is governed by an activated glutamate dehydrogenase. Diabetes, 67(3), 372-384.

4. Anker, M. S., Landmesser, U., von Haehling, S., Butler, J., Coats, A. J., \& Anker, S. D. (2020). Weight loss, malnutrition, and cachexia in COVID-19: facts and numbers. In: Wiley Online Library.

5. Baumgartner, A., Hasenboehler, F., Cantone, J., Hersberger, L., Bargetzi, A., Bargetzi, L., . . Hoess, C. (2021). Effect of nutritional support in patients with lower respiratory tract infection: Secondary analysis of a randomized clinical trial. Clinical nutrition, 4O(4), 1843-1850.

6. Bo, S., Ciccone, G., Castiglione, A., Gambino, R., De Michieli, F., Villois, P., . . Cassader, M. (2013). Antiinflammatory and antioxidant effects of resveratrol in healthy smokers a randomized, double-blind, placebo-controlled, cross-over trial. Current medicinal chemistry, 20(10), 1323-1331.

7. Cengiz, M., Uysal, B. B., Ikitimur, H., Ozcan, E., Islamoğlu, M. S., Aktepe, E., . . Yavuzer, S. (2020). Effect of oral L-Glutamine supplementation on Covid-19 treatment. Clinical nutrition experimental, 33, 24-31.

8. Cetinbas, F., Yelken, B., \& Gulbas, Z. (2010). Role of glutamine administration on cellular immunity after total parenteral nutrition enriched with glutamine in patients with systemic inflammatory response syndrome. Journal of critical care, 25(4), 661. e661-661. e666.

9. Chernyak, B., Popova, E., Prikhodko, A., Grebenchikov, O., Zinovkina, L., \& Zinovkin, R. (2020). COVID19 and oxidative stress. Biochemistry (Moscow), 85(12), 1543-1553.

10. Cruzat, V., Macedo Rogero, M., Noel Keane, K., Curi, R., \& Newsholme, P. (2018). Glutamine: metabolism and immune function, supplementation and clinical translation. Nutrients, 10(11), 1564.

11. Daniel, J. (2020). Education and the COVID-19 pandemic. Prospects, 49(1), 91-96.

12. de Urbina, J. J. O., San-Miguel, B., Vidal-Casariego, A., Crespo, I., Sánchez, D. I., Mauriz, J. L., . . Tuñón, M. J. (2017). Effects of oral glutamine on inflammatory and autophagy responses in cancer patients treated with abdominal radiotherapy: a pilot randomized trial. International journal of medical sciences, 14(11), 1065. 
13. Delgado, T. (2013). Glutamate and GABA in Appetite Regulation. Frontiers in Endocrinology, 4(103). doi:10.3389/fendo.2013.00103

14. Derouiche, S. (2020). Oxidative stress associated with SARS-Cov-2 (COVID-19) increases the severity of the lung disease-a systematic review. J Infect Dis Epidemiol, 6(121).

15. F, F., P, A., \& A, H. (2014). Effects of dietary resveratrol supplementation on liver enzymes, hs-CRP, and hepatic steatosis in patients with nonalcoholic fatty liver disease. ranian Journal of Nutrition Sciences \& FoodTechnology, 8(4), 49-55.

16. Fan, J., Meng, Q., Guo, G., Xie, Y., Xiu, Y., Li, T., ... Ma, L. (2009). Effects of enteral nutrition supplemented with glutamine on intestinal mucosal immunity in burned mice. Nutrition, 25(2), 233239.

17. Hsieh, M.-J., Yang, T.-M., \& Tsai, Y.-H. (2016). Nutritional supplementation in patients with chronic obstructive pulmonary disease. Journal of the Formosan Medical Association, 115(8), 595-601.

18. Iddir, M., Brito, A., Dingeo, G., Fernandez Del Campo, S. S., Samouda, H., La Frano, M. R., \& Bohn, T. (2020). Strengthening the immune system and reducing inflammation and oxidative stress through diet and nutrition: considerations during the COVID-19 crisis. Nutrients, 12(6), 1562.

19. Laforge, M., Elbim, C., Frère, C., Hémadi, M., Massaad, C., Nuss, P., . . Becker, C. (2020). Tissue damage from neutrophil-induced oxidative stress in COVID-19. Nature Reviews Immunology, 20(9), 515-516.

20. Levitt, D. G., \& Levitt, M. D. (2018). A model of blood-ammonia homeostasis based on a quantitative analysis of nitrogen metabolism in the multiple organs involved in the production, catabolism, and excretion of ammonia in humans. Clinical and experimental gastroenterology, 11, 193.

21. Li, Q., Ding, X., Xia, G., Chen, H.-G., Chen, F., Geng, Z., .. Wang, L. (2020). Eosinopenia and elevated Creactive protein facilitate triage of COVID-19 patients in fever clinic: a retrospective case-control study. EClinicalMedicine, 23, 100375.

22. Li, T., Zhang, Y., Gong, C., Wang, J., Liu, B., Shi, L., \& Duan, J. (2020). Prevalence of malnutrition and analysis of related factors in elderly patients with COVID-19 in Wuhan, China. European journal of clinical nutrition, 74(6), 871-875.

23. Liu, N., Huang, R., Baldacchino, T., Sud, A., Sud, K., Khadra, M., \& Kim, J. (2020). Telehealth for Noncritical Patients With Chronic Diseases During the COVID-19 Pandemic. Journal of Medical Internet Research, 22(8), e19493.

24. Moura, C. S., Lollo, P. C. B., Morato, P. N., Risso, E. M., \& Amaya-Farfan, J. (2017). Modulatory effects of arginine, glutamine and branched-chain amino acids on heat shock proteins, immunity and antioxidant response in exercised rats. Food \& function, 8(9), 3228-3238.

25. Naja, F., \& Hamadeh, R. (2020). Nutrition amid the COVID-19 pandemic: a multi-level framework for action. European journal of clinical nutrition, 74(8), 1117-1121.

26. Paladugu, S., \& Donato, A. A. (2020). Remdesivir improved time to recovery in adults hospitalized with COVID-19 and lower respiratory tract involvement. Annals of internal medicine, 173(2), JC4.

27. Patel, A. (2021). Gut-brain Axis Regulation of Food Intake and Visceral IIIness. 
28. Patel, H. J., \& Patel, B. M. (2017). TNF-a and cancer cachexia: Molecular insights and clinical implications. Life sciences, 170, 56-63.

29. Peixoto da Silva, S., Santos, J. M., Costa e Silva, M. P., Gil da Costa, R. M., \& Medeiros, R. (2020). Cancer cachexia and its pathophysiology: Links with sarcopenia, anorexia and asthenia. Journal of cachexia, sarcopenia and muscle, 11(3), 619-635.

30. Qureshi, T., Bjørkmo, M., Nordengen, K., Gundersen, V., Utheim, T. P., Watne, L. O., . . Chaudhry, F. A. (2020). Slc38a1 Conveys Astroglia-Derived Glutamine into GABAergic Interneurons for Neurotransmitter GABA Synthesis. Cells, 9(7), 1686.

31. Rao, S., Schieber, A. M. P., O’Connor, C. P., Leblanc, M., Michel, D., \& Ayres, J. S. (2017). Pathogenmediated inhibition of anorexia promotes host survival and transmission. Cell, 168(3), 503-516. e512.

32. Robinson, P. C., Liew, D. F., Liew, J. W., Monaco, C., Richards, D., Shivakumar, S., ... Feldmann, M. (2020). The Potential for Repurposing Anti-TNF as a Therapy for the Treatment of COVID-19. Med.

33. Rogero, M. M., \& dos Santos, R. V. T. (2017). Glutamine and Exercise and Immune System. In Glutamine (pp. 315-332): CRC Press.

34. Shah, A. M., Wang, Z., \& Ma, J. (2020). Glutamine metabolism and its role in immunity, a comprehensive review. Animals, 10(2), 326.

35. Stehle, P., Ellger, B., Kojic, D., Feuersenger, A., Schneid, C., Stover, J., . . Westphal, M. (2017). Glutamine dipeptide-supplemented parenteral nutrition improves the clinical outcomes of critically ill patients: A systematic evaluation of randomised controlled trials. Clinical nutrition ESPEN, 17, 75-85.

36. Tanha, T., Amanlo, H., \& Fathi, M. (2012). Effcts of Glutamine on Oxidative Stress, Nitrogen Metabolism and Performance of Holestin Dairy Cows in Transition Period. Experimental animal Biology, 1(2), 57-65.

37. Tomé-Carneiro, J., Gonzálvez, M., Larrosa, M., Yáñez-Gascón, M. J., García-Almagro, F. J., Ruiz-Ros, J. A., . . Espín, J. C. (2013). Grape resveratrol increases serum adiponectin and downregulates inflammatory genes in peripheral blood mononuclear cells: a triple-blind, placebo-controlled, one-year clinical trial in patients with stable coronary artery disease. Cardiovascular Drugs and Therapy, 27(1), 37-48.

38. Varela, L., Stutz, B., Song, J. E., Kim, J. G., Liu, Z.-W., Gao, X.-B., \& Horvath, T. L. (2021). Hungerpromoting AgRP neurons trigger an astrocyte-mediated feed-forward autoactivation loop in mice. The Journal of Clinical Investigation, 131(10).

39. Wang, Y., Wang, Q., Li, J., Lu, G., \& Liu, Z. (2019). Glutamine Improves Oxidative Stress through the Wnt3a/ $\beta$-Catenin Signaling Pathway in Alzheimer's Disease In Vitro and In Vivo. BioMed research international, 2019.

40. Yeh, S.-L., Shih, Y.-M., \& Lin, M.-T. (2020). Glutamine and its antioxidative potentials in diabetes. In Diabetes (pp. 255-264): Elsevier.

41. Yuan, S., Zhang, Z.-W., \& Li, Z.-L. (2017). Cell death-autophagy loop and glutamate-glutamine cycle in amyotrophic lateral sclerosis. Frontiers in molecular neuroscience, 10, 231. 


\section{Tables}

Due to technical limitations, tables are only available as a download in the Supplemental Files section.

\section{Supplementary Files}

This is a list of supplementary files associated with this preprint. Click to download.

- Tables.docx 